\title{
Erratum to: Smell Behavior During Odor Preference Decision
}

\author{
Shiori Nakano • Saho Ayabe-Kanamura
}

Published online: 23 August 2013

(C) Springer Science+Business Media New York 2013

\section{Erratum to: Chem. Percept.}

\section{DOI 10.1007/s12078-013-9148-x}

The selectivity and quantity for the experiment 1 in Table 1 were printed mistakenly. The correct table is reprinted below with their legend.

Table 1 Odor stimuli used in each of experiment and selectivity (in percent) of each odor. Selectivity of the intensity group (experiment 2) and the information group (experiment 3 ) are given in parenthesis

\begin{tabular}{|c|c|c|c|c|}
\hline Experiment & Category & Odorants & $\begin{array}{l}\text { Quantity } \\
\text { (g) }\end{array}$ & $\begin{array}{l}\text { Selectivity } \\
(\%)\end{array}$ \\
\hline Experiment 1 & \multirow[t]{7}{*}{ Multiple categories } & Laundry detergent (liquid type: P \& G Japan) & 7.0 & 29 \\
\hline \multirow{6}{*}{$\begin{array}{l}\text { Preference } \\
\quad(n=34)\end{array}$} & & Bar soap (sliced: Cow brand soap KYOSHINSHA Co., Ltd.) & 6.0 & 0 \\
\hline & & Apple (liquid air freshener: S. T. Corporation) & 3.5 & 26 \\
\hline & & $\begin{array}{l}\text { Fragrant olive (solid air freshener: KOBAYASHI Pharmaceutical Co., } \\
\text { Ltd.) }\end{array}$ & 0.2 & 0 \\
\hline & & Grapefruit (essential oil: TREE OF LIFE Co., Ltd.) & 0.1 & 9 \\
\hline & & Peppermint (essential oil: TREE OF LIFE Co., Ltd.) & 0.1 & 29 \\
\hline & & Instant coffee (Nestle Japan) & 1.0 & 6 \\
\hline Experiment 2 & \multirow{6}{*}{$\begin{array}{l}\text { Single category (flavored tea: all } \\
\text { product's brand were LUPICIA Co., } \\
\text { Ltd.) }\end{array}$} & Apple & 7 & $22(5)$ \\
\hline $\begin{array}{r}\text { Preference } \\
(n=23)\end{array}$ & & Peach & 10 & $9(5)$ \\
\hline \multirow{4}{*}{$\begin{array}{c}\text { Intensity } \\
(n=20)\end{array}$} & & Jasmine & 10 & $57(5)$ \\
\hline & & Vanilla & 13 & $0(55)$ \\
\hline & & Chamomile & 8 & $4(20)$ \\
\hline & & Chamomile and orange & $10(3: 7)$ & $9(10)$ \\
\hline Experiment 3 & \multirow{6}{*}{$\begin{array}{l}\text { Single subcategory (Jasmine tea: } \\
\text { six kinds of jasmine tea leaves } \\
\text { selected from different brands) }\end{array}$} & A (Hanamizuki Corporation) & 10 & $70(0)$ \\
\hline $\begin{array}{l}\text { No information } \\
(n=10)\end{array}$ & & B (Ryohin Keikaku Co., Ltd.) & 10 & $0(10)$ \\
\hline \multirow{4}{*}{$\begin{array}{l}\text { Information } \\
(n=10)\end{array}$} & & C (MIKUNIYA Co., Ltd.) & 10 & $10(40)$ \\
\hline & & D (LUPICIA Co., Ltd.) & 10 & $10(10)$ \\
\hline & & E (LUPICIA Co., Ltd.) & 7 & $0(10)$ \\
\hline & & F (ITO EN Ltd.) & 10 & $10(30)$ \\
\hline
\end{tabular}

The online version of the original article can be found at http://dx.doi.org/ 10.1007/s12078-013-9148-x.

S. Nakano $(\bowtie)$

Graduate School of Comprehensive Human Sciences, University of

Tsukuba, 1-1-1 Tennodai, Tsukuba, Ibaraki 305-8572, Japan

e-mail: shionaka@human.tsukuba.ac.jp

\section{S. Ayabe-Kanamura}

Faculty of Human Sciences, Division of Psychology, University of

Tsukuba, 1-1-1 Tennodai, Tsukuba, Ibaraki 305-8572, Japan

e-mail: sahoaya@human.tsukuba.ac.jp 